\title{
Forward Physics at CMS
}

\author{
Roland Benoît for the CMS Collaboration \\ University of Antwerp, Campus Groenenborger, 2020 Antwerp, Belgium
}

\begin{abstract}
.
Studies of forward processes are important tests of the standard model and inputs for Monte Carlo tuning. A measurement of the energy flow in the forward pseudorapidity region of CMS, $3.15<|\eta|<4.9$, is presented for 3 values of the centre-of-mass energy $\sqrt{s}=0.9 \mathrm{TeV}, 2.36 \mathrm{TeV}$ and $7 \mathrm{TeV}$. The forward energy flow is measured for minimum bias events and for events with a central dijet system the transverse energy of which provides a hard scale. The energy flow is compared to various Monte Carlo models with different multiparton interaction schemes. A study of forward jets in the pseudorapidity range $3.2<|\eta|<4.7$ is presented for $\sqrt{s}=7 \mathrm{TeV}$.
\end{abstract}

Keywords: Forward Energy Flow - Forward Jets

PACS: $12.38 . \mathrm{Aw}-13.85 . \mathrm{Hd}-13.87 . \mathrm{Ce}$

\section{MEASUREMENT OF THE FORWARD ENERGY FLOW}

Measurements in the forward region probe the parton content of the proton at small values of the proton momentum fraction, in a region where the parton densities might become very large and where the probability for more than one partonic interaction per event should increase. The measurement of the energy flow in the CMS forward region [1], in the pseudorapidity range $3.15<|\eta|<4.9$, should therefore be sensitive to the modelling of parton radiation at large $\eta$ and the description of multiparton interactions (MPI) [2]. The measurement is performed for two event classes: a minimum bias sample and a sample of events with a central dijet system. The first class is characterized by zero or few partonic interactions, while the second one has at least one hard scattering at the parton level. The amount of parton radiation in the forward region is therefore expected to be larger for the dijet sample, which should be directly reflected in the forward energy flow. The forward measurement can thus provide a complementary and independent constraint on the MPI modelling.

\section{HF calorimeter and Trigger subsystem}

A detailed description of the CMS experiment can be found elsewhere [3] and we only describe here the subsystems used to obtain the presented results. The two Hadronic Forward calorimeters HF+ and HF-, located at $\pm 11.2 \mathrm{~m}$ from the nominal interaction point (IP), cover the pseudorapidity region $2.9<|\eta|<5.2$. These are Cerenkov calorimeters made of radiation hard quartz fibers embedded into steel absorbers. Two subsystems, the Beam Scintillator Counters (BSC) and the Beam Pick-up Timing for the eXperiments (BPTX) are used to trigger the detector readout [4]. The two BSCs are located at $\pm 10.86 \mathrm{~m}$ from the IP and cover the pseudorapidity region $3.23<|\eta|<4$.65. Each is

DIFFRACTION 2010

AIP Conf. Proc. 1350, 168-171 (2011); doi: 10.1063/1.3601400

(C) 2011 American Institute of Physics 978-0-7354-0907-1/\$30.00 
a set of 16 scintillator tiles. The BSC elements have a time resolution of $3 \mathrm{~ns}$ and are designed to provide hit and coincidence rates. The two BPTXs, located around the beam pipe at $\pm 175 \mathrm{~m}$ from the IP, are designed to provide precise information on the bunch structure and timing of the incoming beam, with better than $0.2 \mathrm{~ns}$ time resolution.

\section{Analysis strategy and event selection}

The forward energy flow is measured at 3 values of $\sqrt{s}=0.9 \mathrm{TeV}, 2.36 \mathrm{TeV}$ and $7 \mathrm{TeV}$, for the 2 event classes described above. The following conditions are imposed to select the minimum bias sample. A signal is required in each of the BSCs in conjunction with BPTX signals from both beams passing the IP. This condition rejects a large fraction of diffractive events. A primary vertex is required with $|z|<15 \mathrm{~cm}$ and a transverse distance from the $z$ axis smaller than $2 \mathrm{~cm}$. Further cuts are applied to reject beam-halo event candidates, beam-scraping events and events with large signals consistent with noise in HF. The energy flow is then measured in 5 different $\eta$ bins by summing up all the energy deposits in the HF towers above a noise threshold of $4 \mathrm{GeV}$. The following conditions are imposed to select the dijet sample. Jets are reconstructed by means of the anti- $k_{T}$ jet algorithm [5] with $\mathrm{R}=0.5$. The dijet sample consists of events with at least two leading jets with $|\eta|<2.5$ and $\left|\Delta \phi\left(j_{1}, j_{2}\right)-\pi\right|<1$. Jets are required to have $p_{T}>8 \mathrm{GeV}$ at $\sqrt{s}=0.9 \mathrm{TeV}$ and $2.36 \mathrm{TeV}$, and $p_{T}>20 \mathrm{GeV}$ at $\sqrt{s}=7 \mathrm{TeV}$.

\section{Results and Monte Carlo Comparison}

Figure 1 shows the energy flow in HF at detector level, in the region $3.15<|\eta|<4.9$, for the minimum bias and dijet samples, at $\sqrt{s}=0.9 \mathrm{TeV}, 2.36 \mathrm{TeV}$ and $7 \mathrm{TeV}$. Error bars indicate statistical uncertainties, while the dashed bands represent the total systematic uncertainty. The dominant contribution of $15 \%$ corresponds to the global HF energy scale uncertainty. Detector level distributions are compared to predictions from Monte Carlo event generators passed through the full CMS detector simulation based on Geant 4 [6]. The predictions from PYTHIA6 [7] use different sets of parameters for the MPI: the tunes D6T [8], DW [8], PROQ20 [9] and Perugia P0 [10]. In the latter case a new MPI model is used [11]. The predictions from the Monte Carlo event generators PYTHIA8 [12] and PHOJET [13] are also shown. The forward energy flow in the miminum bias sample shows a stronger energy dependence in data than in Monte Carlo. This behaviour is not observed in the dijet event sample. The energy flow in the $900 \mathrm{GeV}$ minimum bias sample is best described by the D6T tune, whereas the PROQ20 and P0 tunes and PHOJET underestimate the data. At $\sqrt{s}=7 \mathrm{TeV}$, all the Monte Carlo predictions underestimate the data, with PYTHIA8 being close to the PROQ20 tune. In the dijet sample, the D6T tune is too high compared to the data, the P0 tune and PHOJET are too low, and the best description is given by the PROQ20 tune and PYTHIA8. 

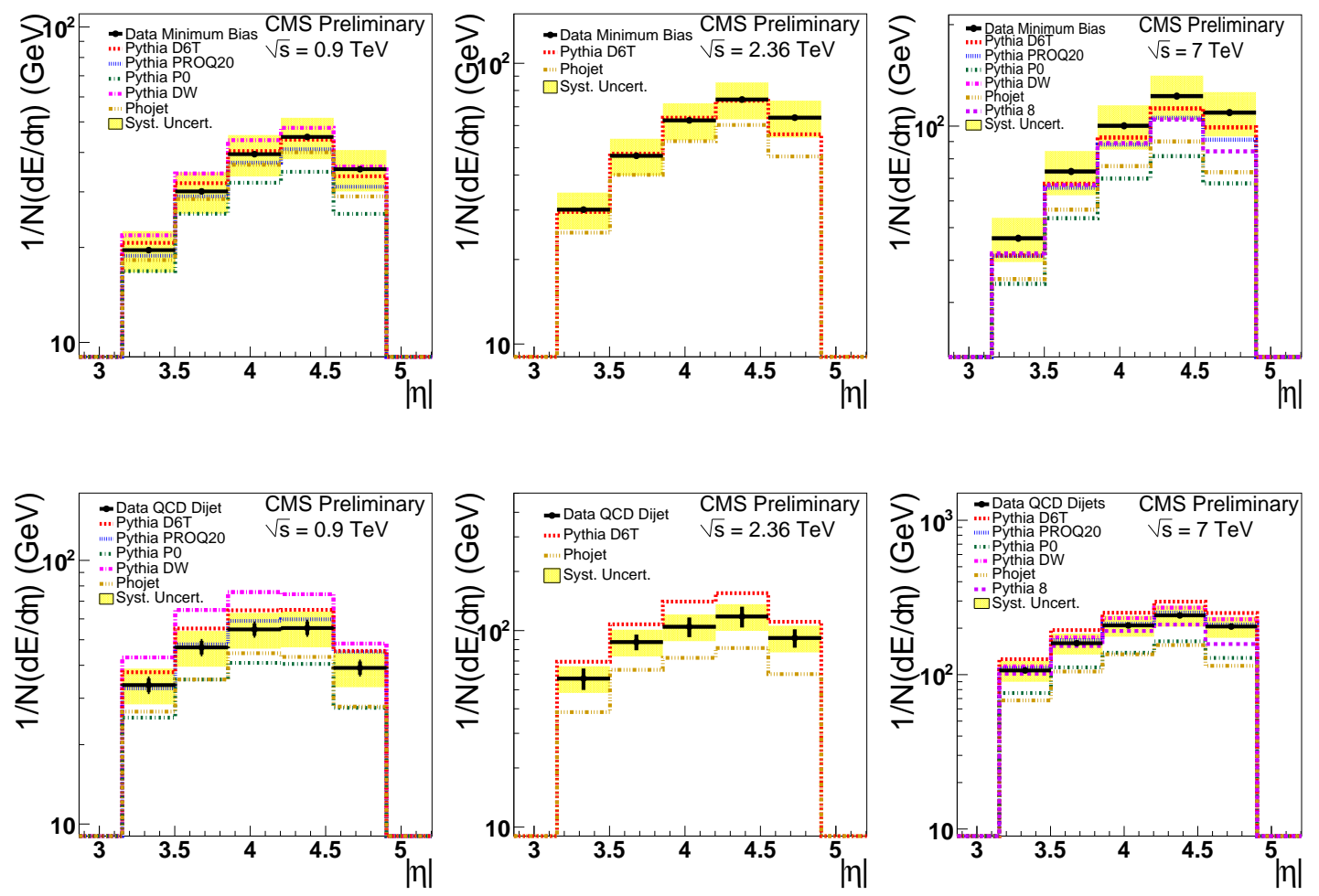

FIGURE 1. Energy flow in the minimum bias sample (top) and dijet sample (bottom) as a function of $\eta$ at $\sqrt{s}=0.9 \mathrm{TeV}$ (left), 2.36 TeV (center) and $7 \mathrm{TeV}$ (right). Detector level distributions are compared to predictions from the Monte Carlo event generators PYTHIA and PHOJET. Error bars correspond to statistical errors. The dashed bands represent the total systematic uncertainty of the measurement.

\section{RECONSTRUCTION OF FORWARD JETS}

Jet production has never been investigated at hadron colliders in such a forward region as the one covered by the HF calorimeter. The first step before measuring forward jets in $\mathrm{HF}$ is to validate the jet reconstruction in that region [14]. The event selection is similar to that used to select the miminum bias sample. Jets are reconstructed by means of the anti- $k_{T}$ jet algorithm [5] with $\mathrm{R}=0.5$, are required to have $35<E_{T}<120 \mathrm{GeV}$ and $3.2<|\eta|<4$.7. The jet energy is corrected for energy loss and non-linear response of the calorimeter. The sample used for the study has been recorded at $\sqrt{s}=7 \mathrm{TeV}$ and corresponds to an integrated luminosity of $10 \mathrm{nb}^{-1}$. Figure 2 shows the distribution of the transverse energy flow $E_{T}$ inside of the jets as a function of the distance $\Delta \eta$ with respect to the jet axis (left), the forward jet $p_{T}$ spectrum (center) and $\eta$ spectrum (right). Detector level distributions with statistical errors only are compared to the predictions of the Monte Carlo event generator PYTHIA6 using the D6T tune. A reasonable agreement is found for the different distributions. 

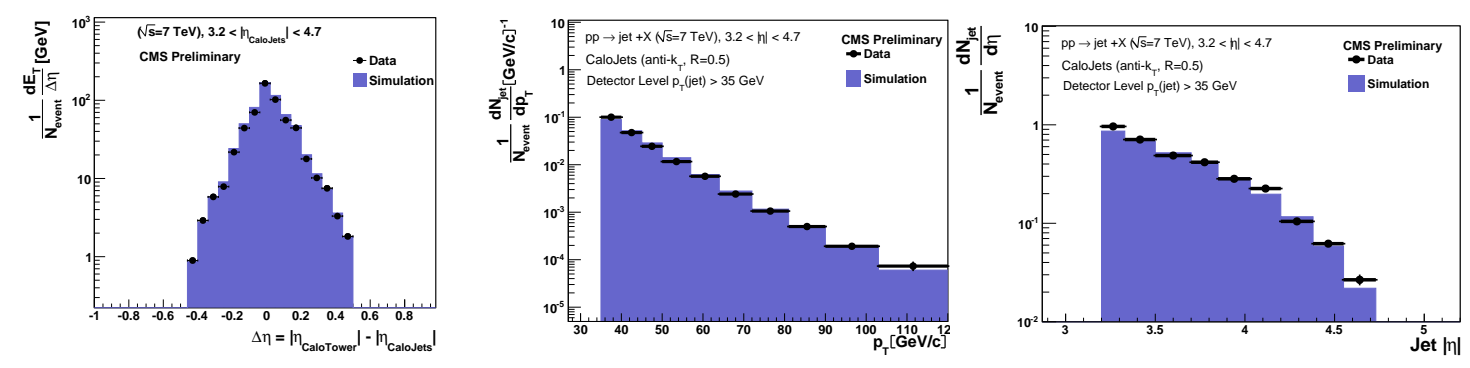

FIGURE 2. The transverse energy flow $E_{T}$ inside of the jets reconstructed in HF as a function of the distance $\Delta \eta$ with respect to the jet axis (left). The forward jet $p_{T}$ spectrum (center) and $\eta$ spectrum (right). Jets are required to have $35<E_{T}<120 \mathrm{GeV}$ and $3.2<|\eta|<4$.7. Detector level distributions are compared to predictions from the Monte Carlo event generator PYTHIA6 using the D6T tune.

\section{CONCLUSION}

The first measurement at hadron colliders of the forward energy flow in the region $3.15<|\eta|<4.9$ has been presented, for a minimum bias sample and a sample of events with a hard scale defined by a central dijet system. The forward energy flow in the miminum bias sample shows a stronger energy dependence in data than in Monte Carlo. At $\sqrt{s}=7 \mathrm{TeV}$, all the Monte Carlo predictions underestimate the measured minimum bias energy flow. Such a behaviour is not observed in the dijet event sample. The Monte Carlo tunes giving the best description in the forward region differ from those giving the best description of the charged particle spectra in the central region [15]. The validation of the forward jets reconstruction in the HF acceptance has been presented at $\sqrt{s}=7 \mathrm{TeV}$.

\section{ACKNOWLEDGMENTS}

I would like to thank my colleagues of the CMS Forward group for the discussions and for providing the results presented in these proceedings.

\section{REFERENCES}

1. CMS Collaboration, PAS-FWD-10-002 (2010).

2. T. Sjostrand and M. van Zijl, Phys. Rev. D 36 (1987) 2019.

3. R. Adolphi et al. [CMS Collaboration], JINST 3 (2008) S08004.

4. T. Aumeyr, Master thesis, Vienna University of Technology, Austria, 2008.

5. M. Cacciari, G. P. Salam and G. Soyez, JHEP 0804 (2008) 063 [arXiv:0802.1189 [hep-ph]].

6. S. Agostinelli et al. [GEANT4 Collaboration], Nucl. Instrum. Meth. A 506 (2003) 250.

7. T. Sjostrand, S. Mrenna and P. Z. Skands, JHEP 0605 (2006) 026 [arXiv:hep-ph/0603175].

8. R. Field, "Studying the Underlying Event at CDF and the LHC," proceedings of the first International Workshop on Multiple Partonic Interactions at the LHC, Perugia, Italy, October 27-31, 2008.

9. A. Buckley, H. Hoeth, H. Lacker, H. Schulz and J. E. von Seggern, Eur. Phys. J. C 65 (2010) 331.

10. P. Z. Skands, "The Perugia Tunes," [arXiv:0905.3418 [hep-ph]].

11. D. Wicke and P. Z. Skands, Nuovo Cim. 123B (2008) S1 [arXiv:0807.3248 [hep-ph]].

12. T. Sjostrand, S. Mrenna and P. Z. Skands, Comput. Phys. Commun. 178 (2008) 852.

13. F. W. Bopp, R. Engel and J. Ranft, arXiv:hep-ph/9803437.

14. CMS Collaboration, DPS-2010-026 (2010).

15. K. Krajczar [CMS Collaboration], PoS E PS-HEP2009 (2009) 062. 
Copyright of AIP Conference Proceedings is the property of American Institute of Physics and its content may not be copied or emailed to multiple sites or posted to a listserv without the copyright holder's express written permission. However, users may print, download, or email articles for individual use. 